\title{
Simulation Application in Mechanics Experiment Research
}

\author{
Xin Zhang ${ }^{1, \text { a }}$ \\ ${ }^{1}$ Baicheng Normal College, Physical and Electronic Information Systems Baicheng, Jilin \\ akzx0001@126.com
}

Keywords: Mechanics Experiments; Simulation Experiments; Computer Simulation

\begin{abstract}
Physics is a branch of science, mainly to explore by experiment. Mechanics is an organic part of physics, mechanics experiment is particularly important. Experiment is a teacher to teach, students learn the important link, is an important part of mechanics.Simulation experiment method in recent years, with the development of network and technology, the foot become a new means of experimental study. Mechanics simulation means starting from the fundamental theorem of mechanics, using the discretization variables to describe the state of the mechanical system, then used a computer computing discrete variables in the evolution laws of mechanics, physical laws and physical methods. This subject mainly introduces the simulation principle, on the basis of further discusses the feasibility of the simulation application in mechanics experiments. Combining the traditional mechanical experiment and simulation experiment so as to improve the effect of learning.
\end{abstract}

\section{1 . Introduction}

\subsection{The Research Background}

Simulation technology is an important technology in modern application in defense. With the development and progress of society, the level of talents needed for the society should be improved。In its report, wealth contains reports that the future can support four kinds of ability to deal with crises: learning to recognize, learn to work, learn to work together, and learn to live ${ }_{\text {In }}$ the new era of information development, we should never be too old to learn. People who do not learn are illiterates, learning becomes a way of living in the future, and losing the ability to learn is both a loss of ability to survive.So, we learn about simulation, and we need to apply the simulation techniques to a broader range of areas.

\subsection{Research Purpose and Significance}

The existing teaching system needs advanced teaching mode. In the study of physics, mechanics is the bridge between the parts, and the knowledge of mechanics is extremely important 。 However, the students who are trained under education are more adept at puzzles and have rich knowledge but can only handle exams. Many experiments in physics are not well understood。 The teaching of demonstration experiment has been completed by the teachers, and some experiments have to achieve the desired effect and consume a lot of resources. So students have a poor grasp of the experiment 。 Therefore, the purpose of this study is to introduce the virtual simulation experiment into the teaching of mechanics experiment, so that some experiments which are not convenient for the actual demonstration can be observed by students.

The simulation experiment led students to immersing themselves in the experiment. The purpose is to train students into all-round talents, not only to master theoretical empty shells, but also to enrich their practical skills.

Under the new curriculum standard, the reform of the teaching process, education is no longer restricted to the teacher's one-man hall, and integrates modern information technology to enrich the color of the classroom. Modern developed technologies are not limited to the mature fields of aerospace, navigation, high-energy weapons, machinery and so on. If they are introduced into the 
frequent education, they can add even greater benefits.

\subsection{Research Content and Research Process}

\section{A.The Research Content}

The research direction of this paper: the application of simulation experiment in mechanics experiment is mainly discussed in the following aspects.

Using computer software to produce an operable multimedia course ware.Advantages of most of the multimedia course ware is intuitive, clear, but some of the physical experiment can only through the simulation, or the way such as building a model to realize its phenomena and results, and the vast majority of course ware without maneuverability, can only watch, can't begin, therefore, operable multimedia course ware becomes the main content of this article.

The experimental results are presented.Full use of multimedia software to simulate the whole experiment process, from demonstration to operation, and can realize the reverse operation and any experimental movement decomposition, the experiment process specific, operational, and complete the whole experiment process and the final result.

Analyze and improve the specific experimental process and results.In the use process, and making of multimedia course ware to discover problems, such as how to realize the maneuverability of the course ware, how to make the experiment process and results more intuitive, more telling, to find a way to improve.

\section{B.The Research Process}

The effective application of the simulation experiment is not shallow, and the whole research process takes the learning simulation concept as the starting point and deeply understands the meaning of the simulation so as to apply it freely to the simulation experiment. In this paper, the application of simulation technology and the various fields of simulation experiment are given. From the simulation tools, simulation software, simulation method, etc., trying to will be introduced to the simulation experiment on mechanics experiment, choose appropriate experiment study of the topic, research the feasibility of the simulation application in mechanics experiments. This paper makes a concrete case practice, and compares the case practice with the analysis of the experiment teaching of ordinary mechanics, and comprehensively considers the advantages and disadvantages of the simulation. To review the research process, summarize the significance of this article.

\subsection{Current Situation Analysis of Domestic and Foreign Simulation System}

\section{A.Foreign Simulation System}

Nowadays many foreign institutions have carried out research and construction of simulation experiment, and there are famous universities or virtual laboratories, which have been put into use of simulation experiment system. As early as 1988, MIT's school of electronic engineering began teaching virtual experiments and was well received by students. In the following time, the university of east Caroline, the university of Houston, and the university of Carolina established virtual laboratories in turn, and the simulation experiment was further developed. The exploration of experimental teaching, the overseas start early, the development fast. Today, we study on simulation experiment is to draw lessons from foreign advanced methods of application of make up for a lack of their own, in combination with the development of the information age, in the domestic make a simulation experiment teaching of the new world.

\section{B.Domestic Research Status}

New technologies such as computers are more widely applied in the physical sciences than other disciplines, leading in other disciplines. By China knowledge network, retrieving "physical and virtual simulation experiment" summary of nearly 10 years related articles can be seen, in physics in recent years the study of simulation experiments total trend is rising, in a paper published in 2011 is more prominent during this period, the journal also increase trend in nearly three years. But the fly in the ointment is that the application of the simulation is not systematic and specific. In this aspect, the research needs to be deepened to learn the advanced aspects of foreign countries. For the application of simulation, this paper only analyzes the study of physical mechanics experiments, and studies and practices. In recent years, the application of patent application in simulation 
experiment has been increasing gradually.

\section{The Simulation Experiment Application Overview}

\subsection{Simulation Experiment Concept}

The computer simulation experiment refers to the multimedia experiment teaching of animation simulation through software. Simulation can simulate many experiment in mechanics, build intuitive visual $2 \mathrm{~d}$ or $3 \mathrm{~d}$ experimental environment, the simulation experiment of simulated reality operation, for students in different space to build virtual real experimental environment. From the laws of physics, experiment methods to experimental operation, to experiment, to deepen understanding the law of experiment, the convenience of the application of the laws of physics in the life, to the laws of physics to students a thorough analysis of the experimental apparatus, experimental equipment interaction experiment project practice. It is more possible to avoid too dangerous subject experiment through the teaching of simulation experiment, and to experiment with higher experimental cost.

\subsection{The Application of Simulation Subject in Teaching}

Reference the application of simulation experiment in the chemical, physical and mechanical experiment of ideal experiments and experimental coefficient of precision requirements, this article is trying to learn predecessors' reference for simulation, the simulation experiment is trying to reference to the mechanics experiments. The time of adjusting the experimental instrument and the process of selecting the ideal experiment result are shortened, and the physical laws revealed by the experimental data are clearly illustrated by the simulation experiment. The teaching of simulation experiment is not limited to the fields of electric engineering, computer, chemistry and electromagnetism, and the wide space development in many fields.

\section{Theoretical Basis}

\subsection{Experimental Features of Physical Mechanics}

Mechanics is the basic part of physical learning, and the earliest contact between middle schools and universities is the mechanical part. From life to physics and from physics to society, physical experiment has broken the original discipline system and built learning framework on the basis of life experience. The physical phenomena of mechanics are most common in life, such as the law of inertia, the force and the reaction. The mechanical experiments are characterized by the combination of movement and movement, the movement of curves and the motion of celestial bodies. In order to study the science and technology, and the further development of machinery, students will learn the mechanics experiment to make more contributions in the future. Second, because mechanical experiment is very common in life, it is easier to stimulate students' interest in learning. Students take interest first, and can apply the rules of the experiment to three applications, verification and deep learning in life.

\subsection{Experiment in the Position of Mechanics}

Study on the earth's orbit around the sun, train turn, free fall, flat throwing motion and other mechanical experiments. It is necessary to demonstrate, to analyze the stress experiment carefully. These mechanical experiments, which depend only on teacher's lecture, are more abstract. It is only by experiment that you can understand the force of the centripetal force, the friction, the air drag, the force of gravity, and the motion of the celestial body cannot be manipulated. By using the simulation experiment, we can observe the good experimental results and avoid the teacher's experiments. These mechanical experiments, only to make, draw out, students can learn to use, flexible through.

Mechanics is not only distributed in the chapters of mechanics, but also in electromagnetism, heat and other courses. For example, the motion of the electron in the magnetic field, the motion of 
the molecular atoms of the heat system, etc. Therefore, it is necessary to clear the physical laws of mechanical experiments in order to learn the mechanics knowledge well. The experiment dominates the study of the study, without experiment, the study of mechanics is like a paper armchair, empty theory, cannot mature application, more alluded to the traditional teaching shortcomings.

\section{4 . Real Experiment and Simulation Experiment}

\subsection{The Meaning and Deficiency of Real Experiment}

In physics, the establishment of physical laws, the definition of physical concepts and the description of physical phenomena are inseparable from the exploration of experiments. The experiment opens the door of physics science, experimental teaching is also the key link in physics teaching, it can be said that the students' thorough understanding of the experiment can make the physics study twice the result. This paper compares two experimental teaching methods, and analyzes their advantages and disadvantages as follows:

\section{A.The Meaning of Real Experiment}

The first is that the real experiment can cultivate students' perceptual knowledge. They all say that seeing is better than seeing. Real hands-on try, can from the perceptual system of the students, to actually feel the presence of physical laws, through the experiment of can see the effect, the understanding of the physical world is more apparent.

Secondly, it is easy for students to focus on the new things. Although the physical phenomenon is common in life, there are many small experiments that use the laws of physics, and experiments that apply the laws of physics must attract students. This improves the student's interest in learning, focuses on the attention of the study, and achieves better teaching quality.

\section{B. The Lack of Real Experiments}

The first is that the actual experiment can not accurately predict the results of the experiment. The error of the experimental results is very large, which will affect the students' acceptance of the laws of physics, which will not only result in poor teaching effect, but also affect the teaching efficiency and waste class time.

Secondly, the student learning experiment may need to be watched repeatedly to avoid missing the experimental procedure. But the real experiment is done only once, the student wants to study the experiment in the class is very difficult to carry on, the convenience sex is poor, causes the student not to study immediately, the profound understanding principle and grasps the experimental method.

Third, not all experimental equipment can be prepared, and some experiments may not be carried out under the constraints of school funding. This brings us back to the experimental ground teaching, which limits the effectiveness of teaching and opens the gap between students in different regions.

\subsection{The Advantages and Limitations of the Simulation Experiment}

\section{A.The Advantages of Simulation Experiment}

Due to factors such as environment, equipment, space, security restrictions, many important physical experiment can't be, let the students in the experimental teaching completed personally only through demonstration experiment teaching means, lounge or computer demonstration. For example: the operation of the satellite and the planets, the basic particles, the electrons, the neutrons movement, the radium elements, etc. Physics experiment can not only went up from the surface observation, the physical laws exist, even on the number of precise calculation, in this way, TV, video and other means is dull, can't calculate, not arbitrary simulation, only the modern computer multimedia technology can put the TV, telephone, computer, using digital signal technology, demonstrate the simulation of various physical and mechanical experiment.

\section{B.Limitations of the Simulation Experiment}

Now some scholars at home and abroad in computer simulation of physics experimental have done some research, mainly solved how to make use of individual can't intuitive present physical experiment, computer simulation and the simulation process, and make a deep analysis method, but 
for the present computer in physics experiment operation is still an open question. Although the simulation experiment can demonstrate the difficult experiment of practical operation, the students can not do experiments with their own hands, and the perception effect of the experiment is greatly reduced.

Teachers can't understand students' mastery of the experiment by observing their own hands-on experiment. Not conducive to training students to solve practical problems; Simulation experiments, after all, are experiments in virtual environments that do not provide an objective perception. The experimental results of the simulation experiment point to the clear, lack of the problem of the random experiment and the result of the experiment, which is not conducive to the cultivation of the ability to solve the experimental problem.

\section{Conclusion}

In the context of the present era, the development history and development status of the simulation experiment are analyzed. Combining with the characteristics of today's mechanical experiment, the simulation experiment is applied to the mechanical experiment, and the significance of the simulation experiment is revealed deeply. Analyze the concept of simulation and clarify the purpose of this study. The purpose is clear and clear, considering the insufficiency of the real experiment, which is replaced by the simulation experiment. The rational cognition simulation experiment is not perfect, the development of the simulation should be perfected in the future, and the history of the simulation should be witnessed. Example of proof mechanics, the system carries out the simulation thought.

\section{References}

[1]Wang Xiaobo,Hou Jianqing,Guo Yugang.University physical simulation experiment and teaching practice [J]. Physics Experiment,2001(01);

[2]Ning Xin, Pang Wei, Chen Jun.The application of college physics simulation experiment in teaching practice [J]. University Physics Experiment,2012(03);

[3]Huang Zhongnong. The role of simulation experiment in physics experiment teaching [J]. China Science and Technology Innovation Guide,2012(10);

[4]Luo Shouheng. Physics experiments and physical simulation experiments [J]. Journal of Natural Sciences, 2000 (09);

[5]Liu Liping. The application of simulation physics experiment in high school physics teaching [J]. The Teacher's Principle,2013 (11);

[6]Hou Yantao,Wang Xiaolin.Simulation results applied to the principles of chemical engineering teaching thinking [J]. Science and Technology Horizon;2014 (13). 